\title{
PRESSURE SHIFTS OF METAL LINES IN COOL WHITE DWARFS
}

\author{
Gordon L. Hammond \\ Astronomy Program, Department of Mathematics \\ University of South Florida, Tampa, FL $336 z \emptyset$
}

\section{Abstract}

Model atmosphere techniques and an experimentally confirmed theory of collision broadening of $\mathrm{CaII} H$ and $\mathrm{K}$ by helium have been combined to show that the difference in velocity shifts for the two lines is a sensitive diagnostic for the $\mathrm{H} / \mathrm{He}$ abundance ratio in cool, metallic line white dwarfs.

\section{Introduction}

Greenstein (1972) discussed the role of pressure shifts in his analysis of the radial velocity of the DZ8 van Maanen 2. He measured a discrepancy of $29 \mathrm{~km} / \mathrm{s}$ in velocity of the two fine structure components, $H$ and $K$, of CaII. Now that high resolution spectra of $D Z$ stars are becoming available (see, for example, Wagner, Sion, Liebert and Starrfield 1988; Liebert, Wehrse and Green 1987), and the theoretical approach to neutral collision broadening has recently improved beyond the classical treatments of van der Waals and Lennard-Jones interaction potentials, a more precise study of pressure shifts in these high density atmospheres seems warranted.

The goals of this work are to provide corrections to observed radial velocities for more accurate gravitational redshift determinations, and to determine if the fine structure shift behavior might provide evidence of the H/He ratios. This evidence can be crucial in studies of accretion hypotheses (e.g., Aannestad and Sion 1985; Zeidler, Weidemann and Koester 1986).

\section{Collision Broadening and Shifts}

Laboratory measurements of both broadening and shifts of $C$ aII $H$ and $K$ by helium under white dwarf atmospheric conditions were made by Hammond (1975). Those damping constants were used in a white dwarf model atmosphere program (Hammond 1974), but the experimental shifts were small and the temperature range was too small for accurate determination of the temperature dependences.

A recent theoretical study of the perturbation of both $H$ and $K 1$ ines of Call by helium collisions was made by Monteiro, Cooper, Dickinson and Lewis (1986) using model potential methods. That uork predicts broadening and shift parameters under impact approximation conditions, and the temperature dependence of these quantities. 
The excellent agreement between experiment and theory for the damping constants has already beer described (Hammond 1987). The agreement for shifts is shown in Figure 1, where least squares fits to both theory plus experiment (a), and to theory alone (b), are shown. Aside from some numerical noise in

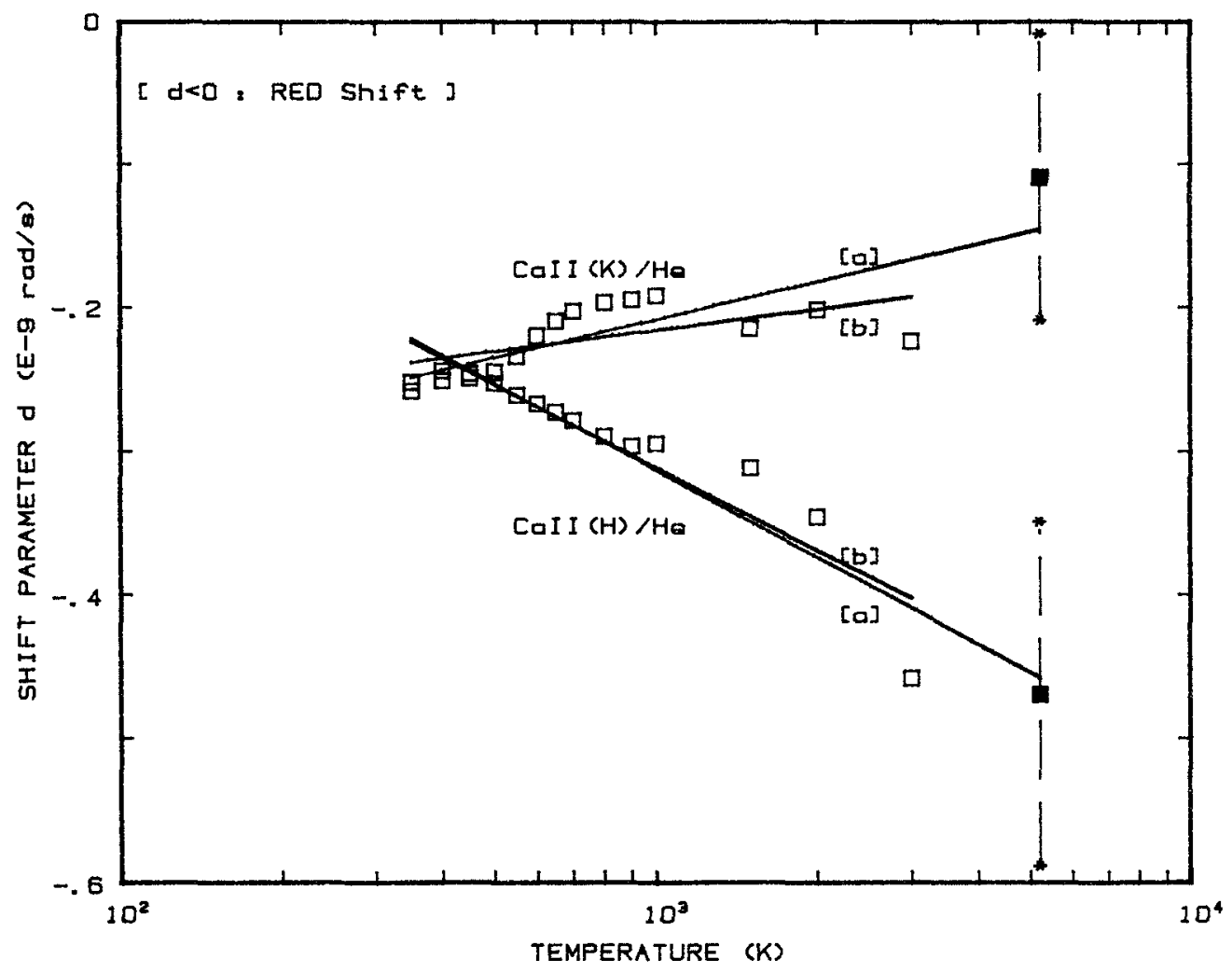

Fig. 1-- Temperature dependence of the CaII H \& K line shift parameters. The experimental points (filled squares) show the probable errors from nine measurements at an average temperature of $5200 \mathrm{~K}$.

the theoretical points (open squares), there is good agreement in magnitude, temperature dependence, and shift direction. The fits for theoretical plus experimental points, (a), have been added to the model atmosphere code.

\section{Model Atmosphere Program}

The early form of the code was written by Bell (1970) for work on $G$ and $K$ giants. Hammond (1974) modified it to treat high gravity stars, paying close attention to obtaining flux constancy in the presence of strong convection. The program yields flux-constant, non-grey, LTE models, and calculates UBVRI and uvby colors and several MCSP colors (Greenstein 1984). The H and K line 


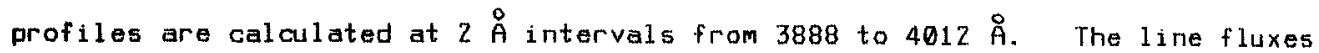
are convolved with an instrumental slit function of $4 \AA$ half-width. The wavelength shifts are measured by fitting a trigonometric series expansion to the line cores. The wavelength of the bisector of the core can thus be determined to $0.05 \AA$ 品 $(4 \mathrm{~km} / \mathrm{s})$. This technique simulates the Greenstein Grant machine method used on photographic spectra, and is applicable to electronic spectra where noise at the minima also reduces precision. The fitting function also can fit the asymmetric profiles exhibited by the cool, helium rich models.

\section{Results and Discussion}

Inspection of Fig. 1 indicates the $H$ line shifts much more than the $K$ line at temperatures greater then $1000 K$, and the difference in shift, $V(H)-V(K)$, will then be a function of atmospheric pressure. That pressure, in turn, will be a function of the H/He ratio due to the differing opacities of $\mathrm{H}^{-}$ and $\mathrm{He}^{-}$. It only remains to determine the magnitude of the differential shift.

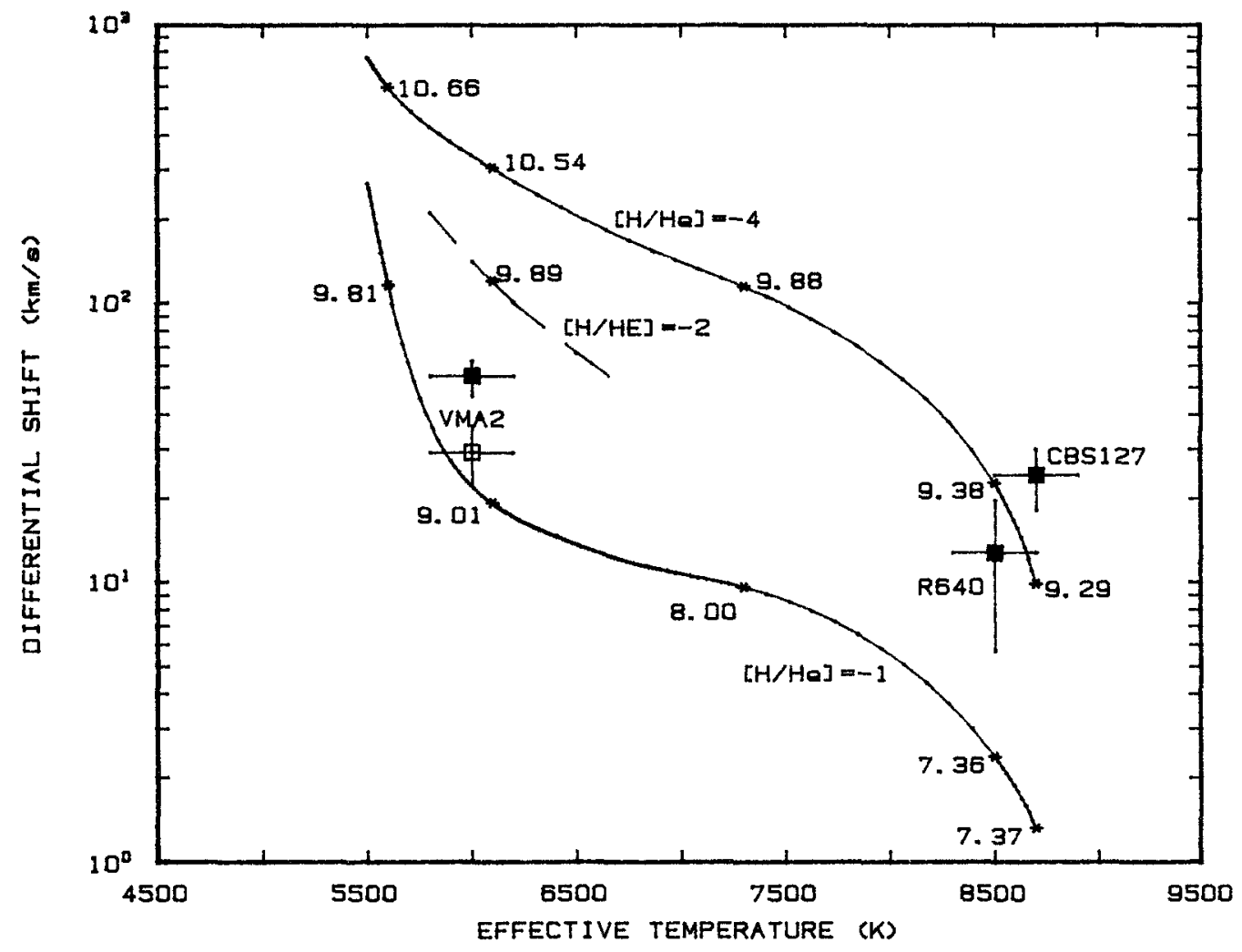

Fig. 2-- Differential shifts from the model grid and observational data. Points (*) on the curves are labeled with $109 P_{0}$ at tau= 0.10 . The filled squares are from data supplied by Sion (1988); the open square is from Greenstein (1972). 
The results from a grid of models with effective temperatures from $5600 \mathrm{~K}$ to $8700 \mathrm{~K}$ and $\mathrm{H} / \mathrm{He}$ abundance ratios from $10 \%$ to $0.01 \%$ are shown in Figure 2 . All models were calculated with $109 g=8.0$, and the metal abundances were adjusted to yield equivalent widths of $H$ plus $K$ of about $35 \AA$, a value typical of cool DZ stars. The upper 1 imit of $10 \%$ hydrogen was dictated by the lack of data on the shifts of $H$ and $K$ by hydrogen collisions. The grid models are labeled with $\log \mathrm{Pg}$ at tau $=0.10$; the curves are empirical cubic spline fits.

There is a clear separation of the helium rich models from the hydrogen contaminated models; the differential shift increases by about 1 dex for the helium rich models at any effective temperature. At differential shifts greater than about $50 \mathrm{~km} / \mathrm{s}$, the core of the H line becomes noticeably red asymmetric due to the high gas pressures and large shift parameters. This is also the regime where the impact approximation becomes invalid for the line wings (helium number densities greater than $5 . E+21 / \mathrm{cc}$ ), and the regime where departures from the ideal gas equation of state may become important. It remains to be seen whether any real $O Z$ stars exhibit such large differential shifts and asymmetry.

The differential shift for vMa2 (open square, Greenstein 1972) and for several $D Z$ stars with high resolution electronic spectra (filled squares, Sion 1988) are shown in Fig. 2 ; the effective temperatures are from matching model and observed colors and continuum fluxes. The agreement between the old photographic measurements and the new data for VMa2 is good and indicates a H/He ratio between $1 \%$ and $10 \%$, a result that agrees with a determination from infrared colors (Hammond 1974). The result for Ross 640, $10 \mathrm{~g}(\mathrm{H} / \mathrm{He}$ ) $=-4$, agrees with the hydrogen abundance found by Liebert (1977) from very weak Balmer lines. Several other cool $D Z$ stars with strong $H$ and $K$ lines have been observed at high resolution by Sion (1988) and are now being analyzed in the above fashion. The present results show that the differential shift, coupled with accurate effective temperatures, can reveal the H/He abundance ratio in cool DZ stars within quite narrow limits.

\section{References}

Aannestad, P. A., and Sion, E. M. 1985, A. J.,90,1832.

Bel1, R. A. 1970, M.N.R.A.S., 148,25.

Greenstein, J. L. 1972, Ap. J., 173,377 .

Greenstein, J. L. 1984, Ap. J. ,276, 602.

Hammond, G. L. 1974, Ph.D. Dissertation, University Maryland, College Park, MD

Hammond, G. L. 1975, Ap. J. 196, 291 .

Hammond, G. L. 1987, Bull. ÁAS, 19,755 .

Liebert, J. 1977, Ástr. Ap. BQ, 101 .

Liebert, J., Wehrse, R., andGreen, R. F. 1987, Astr. Ap. $175,173$.

Monteiro, T. Cooper, I', Dickinson, A., and Lewis, E. 1986, J. Phys. B, 19, 4087 Sion, E. M. 'ig88, private communicatión.

Wagner, R. Sion, E. M., Liebert, J., and Starrfield, S. 1988, Ap. J. $328,213$.

Zeidler-K.t., E.-M., Weidemann, V., and Koester, D. 1986, Astr. Ap., $1555,356$. 